\title{
CLINICAL RESEARCH ARTICLE Prolonged duration of early antibiotic therapy in extremely premature infants
}

Rachel G. Greenberg (1)', Dhuly Chowdhury ${ }^{2}$, Nellie I. Hansen ${ }^{2}$, P. Brian Smith ${ }^{1}$, Barbara J. Stoll ${ }^{3}$, Pablo J. Sánchez ${ }^{4}$, Abhik Das ${ }^{5}$, Karen M. Puopolo ${ }^{6}$, Sagori Mukhopadhyay ${ }^{6}$, Rosemary D. Higgins ${ }^{7}$ and C. Michael Cotten ${ }^{1}$ for the Eunice Kennedy Shriver National Institute of Child Health and Human Development Neonatal Research Network

BACKGROUND: Prolonged early antibiotics in extremely premature infants may have negative effects. We aimed to assess prevalence and outcomes of provision of prolonged early antibiotics to extremely premature infants in the absence of cultureconfirmed infection or NEC.

METHODS: Cohort study of infants from 13 centers born without a major birth defect from 2008-2014 who were 401-1000 grams birth weight, 22-28 weeks gestation, and survived $\geq 5$ days without culture-confirmed infection, NEC, or spontaneous intestinal perforation. We determined the proportion of infants who received prolonged early antibiotics, defined as $\geq 5$ days of antibiotic therapy started at $\leq 72 \mathrm{~h}$ of age, by center and over time. Associations between prolonged early antibiotics and adverse outcomes were assessed using multivariable logistic regression.

RESULTS: A total of 5730 infants were included. The proportion of infants receiving prolonged early antibiotics varied from $30-69 \%$ among centers and declined from $49 \%$ in 2008 to $35 \%$ in 2014. Prolonged early antibiotics was not significantly associated with death (adjusted odds ratio 1.17 [95\% Cl: 0.99-1.40], $p=0.07$ ) and was not associated with NEC.

CONCLUSIONS: The proportion of extremely premature infants receiving prolonged early antibiotics decreased, but significant center variation persists. Prolonged early antibiotics were not significantly associated with increased odds of death or NEC.

Pediatric Research (2019) 85:994-1000; https://doi.org/10.1038/s41390-019-0300-4

\section{INTRODUCTION}

Antibiotics are the most common medications prescribed for infants in the neonatal intensive care unit (NICU). ${ }^{1}$ Although the incidence of culture-confirmed early-onset sepsis (EOS) in term infants is relatively low ( 0.5-0.8 cases/1000 live births), both the incidence of disease (15-19 cases/1000) and EOS-attributable mortality (3.5\% vs $35 \%)$ are substantially higher among very low birth weight, preterm infants. ${ }^{2}$ Given the high mortality associated with EOS, presence of maternal and neonatal risk factors, and time required for pathogens to be isolated in culture, empirical antibiotics targeting EOS are administered to large proportions of infants in the NICU. ${ }^{3,4}$

In 2009, the Eunice Kennedy Shriver National Institute of Child Health and Human Development Neonatal Research Network (NRN) reported data from 1998 to 2001 suggesting that prolonged duration of antibiotics administered soon after birth to extremely low birth weight (ELBW, birth weight $<1000 \mathrm{~g}$ ) infants was associated with increased risk of death, necrotizing enterocolitis (NEC), and the composite measure of NEC or death. ${ }^{5}$ In that study, $53 \%$ of 4039 infants surviving $>5$ days were administered antibiotics beginning at birth and extending $\geq 5$ days in the absence of culture-confirmed infection. These findings were consistent with other smaller studies. ${ }^{6,7}$ It is unclear to what degree neonatal clinicians have changed antibiotic practice over the ensuing 20 years and the impact on infant outcomes. A recent study including 40,534 very low birth weight (VLBW, <1500 g) infants born at 297 primarily non-teaching centers found a small but significant decrease in the rate of prolonged early antibiotic administration among VLBW infants, but no change among the subgroup of 14,923 ELBW infants. ${ }^{8}$ The purpose of this study was to (1) describe changes over time in the provision of prolonged early antibiotic therapy without culture-confirmed infection or intraabdominal inflammatory process, and (2) describe associations of such prolonged early antibiotic therapy with adverse outcomes among a more contemporary cohort of extremely premature infants cared for at academic centers of the NRN.

\section{MATERIALS AND METHODS}

Subjects

This was a retrospective analysis of data entered into a registry of high-risk preterm infants maintained by the NRN. Study infants were born between January 1, 2008, and December 31, 2014. The registry contains maternal and neonatal information from the intrapartum period until infant discharge home, transfer from the primary center, death, or 120 days after birth. Limited

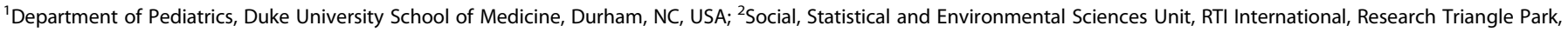

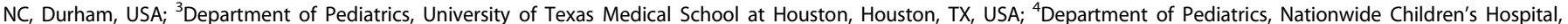

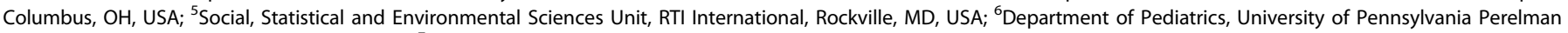

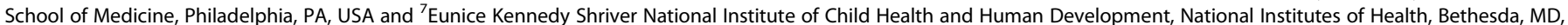
USA

Correspondence: Rachel G. Greenberg (rachel.greenberg@duke.edu)

Received: 12 November 2018 Revised: 4 January 2019 Accepted: 8 January 2019

Published online: 22 January 2019 
demographic, clinical, and outcome data, including whether or not infants were started on antibiotics before and continued beyond the first 5 postnatal days, were collected by trained research staff utilizing a standardized manual of operations. Site participation differed over the study period, and we included data from the 13 centers that participated in the NRN for the entire study period (denoted with an asterisk in the Acknowledgments). Infants were included in the study if they had a birth weight of 401-1000 $\mathrm{g}$ and gestational age of 22 0/7-28 6/7 weeks. Exclusion criteria were: death $<5$ days after birth; blood or cerebrospinal fluid culture obtained within the first 5 postnatal days growing pathogenic bacterial or fungal species; NEC or spontaneous intestinal perforation (SIP) diagnosed $\leq 5$ days after birth; or major birth defect. The institutional review board at each center approved participation in the registry.

\section{Definitions}

Prolonged early antibiotic therapy was defined as $\geq 5$ days of antibiotic administration initiated at $\leq 72 \mathrm{~h}$ of age. Comparator infants may have had antibiotics initiated within $72 \mathrm{~h}$ of age and discontinued before 5 days, or may not have had antibiotics initiated within $72 \mathrm{~h}$ of age. Small for gestational age (SGA) was defined as birth weight $<10$ th percentile for sex and gestational age using percentiles reported by Alexander et al. ${ }^{9}$ Late-onset sepsis (LOS) was defined as a blood and/or cerebrospinal fluid culture obtained at $>72 \mathrm{~h}$ of age and growing a recognized bacterial or fungal pathogen if the infant was administered antibiotics for $\geq 5$ days or until death. NEC was defined as modified Bell's stage $\geq I I A{ }^{10,11}$

\section{Statistical analysis}

Prior to excluding infants who died at $<5$ days or who had a positive culture, NEC, or SIP in the first 5 days, we determined the proportion of infants with EOS by center. After exclusions, we determined the proportion of infants who received prolonged early antibiotics overall and by center over time. We compared infant demographic and maternal characteristics between infants who received prolonged early antibiotics and those who did not using Student's $t$-test for continuous variables and Wald $x^{2}$ test for categorical variables. $p$-values were two-tailed with values $\leq 0.05$ considered statistically significant. Analyses were conducted using SAS 9.4 (SAS Institute, Cary, NC).

Separate logistic regression models were used to evaluate associations between birth year, center, and receipt of prolonged early antibiotics; and between prolonged early antibiotics and outcomes, including all-cause mortality after 5 days of life, NEC, LOS, LOS due to organisms other than coagulase-negative Staphylococci (non-CoNS LOS), and LOS due to one or more fungal organisms (fungal LOS). We examined death and composite outcomes of death/morbidity for all infants; NEC and LOS were examined in survivors to discharge to reduce potential bias due to different at-risk periods, particularly for infants with short survival times. All models included birth year (categorical), study center, infant characteristics (gestational age, SGA, sex, and 5-min Apgar score $<5$ ), and maternal characteristics (black race, multiple birth, rupture of membranes $>24 \mathrm{~h}$, receipt of antenatal steroids, perinatal antibiotic therapy, hypertension, antepartum hemorrhage, and cesarean section).

Because prolonged early antibiotics may be administered to more severely ill infants at greater risk for adverse outcomes, we also included an indicator in our models aimed at identifying severity of illness. We defined this indicator as initiation of any mechanical ventilation on the day of birth and continued use for all of the first 7 days after birth, or for all days until death if death occurred before 7 days (continuous mechanical ventilation [CMV]). We examined whether associations between prolonged early antibiotics and adverse outcomes were different among infants with and without this indicator by including the interaction between prolonged early antibiotics and CMV in our models. Results are shown separately for the $\mathrm{CMV}$ and non-cMV groups if this interaction was significant at $p \leq 0.10$. Otherwise, results shown are based on models that did not include the cMV interaction.

\section{RESULTS}

The NRN registry enrolled 14,212 infants from 2008 to 2014. Of these, 5730 met all study inclusion and exclusion criteria (Supplemental Digital Content S1). Prolonged early antibiotics were administered to $2526 / 5730$ (44\%) infants. Multiple maternal and neonatal characteristics differed significantly between infants who did or did not receive prolonged early antibiotics (Table 1). Overall, infants who received prolonged early antibiotics were younger and smaller, had lower 5-min Apgar scores, were less likely to have been born via cesarean section, were more likely to have been born to a mother with prolonged rupture of membranes or who was treated with antenatal antibiotics, and were more likely treated with CMV (Table 1).

Factors associated with receipt of prolonged early antibiotics The overall percentage of infants treated with prolonged early antibiotics decreased from $49 \%$ in 2008 to $35 \%$ in 2014 (adjusted odds ratio [aOR] 0.44 [95\% Cl: 0.35-0.56], $p<0.001$ ) (Supplemental Digital Content S2, Table 2). The percentage of infants treated varied by center, ranging from 30 to $69 \%$ (Fig. 1). The centerspecific incidence of EOS ranged from 0.3 to $3.6 \%$. The centerspecific rate of prolonged early antibiotic administration decreased over the study period at 8 of the 13 centers. Compared to the center with the lowest rate, infants cared for at 11 of the 12 remaining centers were significantly more likely to receive prolonged early antibiotics (Table 2 ).

Relationship of prolonged early antibiotics with outcomes Infants who received prolonged early antibiotics had marginally but not significantly higher odds of death compared to those who did not receive prolonged early antibiotics (19\% vs 13\%, aOR 1.17 [95\% Cl: 0.99-1.40], $p=0.07$ ). Prolonged early antibiotics were not significantly associated with the composite outcomes of death or specific morbidities (Table 3), nor with specific morbidities among survivors to discharge. These findings were not different among infants who did or did not receive CMV, with one exception: the relationship of prolonged early antibiotics with LOS caused by fungal organisms varied by CMV group (interaction $p<0.001$ ) (Table 3 ). Among infants not treated with $\mathrm{CMV}$, prolonged early antibiotic administration was associated with increased odds of fungal LOS ( $2 \%$ vs $0.4 \%$, aOR 4.95 [95\% Cl: 1.93-12.69], $p<0.001$ ) (Table 3).

\section{DISCUSSION}

We observed a significant decline in the overall use of prolonged early antibiotics among ELBW infants cared for in 13 NRN centers over a 7-year period. The overall use of prolonged early antibiotic therapy during this study was lower (44\% vs 53\%) than that reported in our prior study of early antibiotic use in infants born 1998-2001. However, we observed persistent significant centerspecific antibiotic practice variation. The proportion of infants treated with prolonged early antibiotics differed between centers, and reduction in prolonged therapy was only observed at 8 of the 13 centers. The rates of prolonged antibiotic administration remained 10-to-100-fold higher than the rates of cultureconfirmed EOS, even with the observed decrease in prolonged therapy. In contrast to our prior NRN study, we found no statistically significant association between prolonged early antibiotics and risk of death. Our study raises several issues to be considered as neonatal caregivers work to identify the best 
Table 1. Demographics and clinical characteristics

\begin{tabular}{|c|c|c|c|}
\hline & Prolonged early antibiotics $(N=2526)$ & No prolonged early antibiotics $(N=3204)$ & $p$-value \\
\hline \multicolumn{4}{|l|}{ Infant characteristics } \\
\hline Birth weight, g (median, IQR) & $730(615,850)$ & $790(670,890)$ & $<0.001$ \\
\hline By gestational age week & & & $<0.001$ \\
\hline 22 & $1 \%$ & $1 \%$ & \\
\hline 25 & $24 \%$ & $21 \%$ & \\
\hline 26 & $20 \%$ & $23 \%$ & \\
\hline 27 & $13 \%$ & $21 \%$ & \\
\hline 28 & $7 \%$ & $14 \%$ & \\
\hline Small for gestational age & $11 \%$ & $11 \%$ & 0.48 \\
\hline Race/ethnicity ${ }^{\mathrm{b}}$ & & & 0.19 \\
\hline Black & $45 \%$ & $43 \%$ & \\
\hline White & $36 \%$ & $38 \%$ & \\
\hline Hispanic & $13 \%$ & $14 \%$ & \\
\hline Other & $6 \%$ & $5 \%$ & \\
\hline Multiple birth & $23 \%$ & $26 \%$ & 0.01 \\
\hline Hypertension & $26 \%$ & $37 \%$ & $<0.001$ \\
\hline Antenatal steroids & $89 \%$ & $91 \%$ & 0.05 \\
\hline Antenatal antibiotics & $76 \%$ & $71 \%$ & $<0.001$ \\
\hline Rupture of membranes $>24 \mathrm{~h}$ & $28 \%$ & $18 \%$ & $<0.001$ \\
\hline
\end{tabular}

Information was missing as follows: maternal race/ethnicity, 16 infants; maternal hypertension, 8 infants; antepartum hemorrhage, 5 infants; antenatal steroids, 12 infants; antenatal antibiotics, 34 infants; timing of rupture of membranes, 159 infants; cesarean delivery, 3 infants; 5-min Apgar score, 14 infants; mechanical ventilation, 1 infant

$I Q R$ Interquartile range

${ }^{\text {a }}$ This group includes 40 infants who were intubated continuously until death before day 7

${ }^{b}$ Maternal race/ethnicity was defined as non-Hispanic white, non-Hispanic black, Hispanic (both white and black), and other race (Asian/Pacific Islander, American Indian/Alaska native, more than one race, other not specified). Those of white or black race with missing ethnicity information ( $5.3 \%$ of blacks, $0.9 \%$ of whites) were classified as non-Hispanic

approaches to use of antibiotics for medically fragile, extremely premature infants in the first postnatal days.

Our findings reflect slowly evolving views of the role of antibiotics in neonatal care. Multiple national organizations recommend comprehensive approaches to antimicrobial stewardship, ${ }^{12}$ and neonatal providers are actively pursuing approaches to optimize antibiotic use among NICU patients. ${ }^{13}$ In a study using the Pediatric Health Information System database, antibiotic utilization decreased $~ 5 \%$ per year from 2004 to 2012 among hospitals without mandatory public reporting requirements for central line-associated bloodstream infection; ${ }^{14}$ similar secular trends in NICU antibiotic use were observed among hospitals subject to such reporting requirements. Neonatal antimicrobial stewardship programs at individual centers and collaboratives also report success in safely decreasing overall NICU antibiotic utilization. ${ }^{15-17}$ However, NICUs care for infants across a wide spectrum of gestational ages and morbidities, and studies focused specifically on VLBW and ELBW infants report mixed outcomes. The Premier Perspective Database study measured quarterly rates of antibiotic initiation and duration among 40,364 VLBW infants cared for in 297 centers across the United States from 2009 to
2015. ${ }^{8}$ No significant trends were observed for antibiotic initiation, but declines were noted for prolonged early antibiotics among VLBW (but not ELBW) infants. The Canadian Neonatal Network (CNN) reported decreased antibiotic use in VLBW infants between 2010 and 2014, a finding that coincided with decreased rates of LOS. $^{18}$ Despite this decline, the study still found an overall $20 \%$ antibiotic utilization rate among VLBW infants in the absence of culture-confirmed infection or NEC. Single-site studies further illustrate the challenges of antibiotic optimization among premature infants. Guidelines for use of antibiotics targeting highly antibiotic-resistant pathogens (e.g., meropenem, vancomycin) resulted in more appropriate antibiotic use in one Canadian NICU, but improvements were not seen among VLBW infants. ${ }^{19} \mathrm{~A}$ comprehensive antibiotic stewardship program in a large American NICU did not significantly decrease overall antibiotic utilization, although some changes were observed in the use of specific antibiotics such as ampicillin and vancomycin. ${ }^{20}$ Viewed from the perspective of the many challenges in optimizing neonatal antibiotic administration, the decline in prolonged early antibiotic administration we observed in the current study $(14 \%$ absolute change, $28 \%$ relative change) among ELBW infants 
Table 2. Differences in receipt of prolonged early antibiotic therapy by birth year and center adjusting for neonatal and maternal characteristics

\begin{tabular}{|c|c|c|}
\hline & $\begin{array}{l}\text { Adjusted odds ratio (95\% } \\
\text { confidence interval) }\end{array}$ & $p$-value \\
\hline \multicolumn{3}{|l|}{ Birth year (reference: 2008) } \\
\hline 2009 & $0.89(0.72-1.10)$ & 0.29 \\
\hline 2010 & $1.02(0.82-1.27)$ & 0.83 \\
\hline 2011 & $0.79(0.63-0.98)$ & 0.03 \\
\hline 2012 & $0.58(0.47-0.72)$ & $<0.001$ \\
\hline 2013 & $0.58(0.47-0.73)$ & $<0.001$ \\
\hline 2014 & $0.44(0.35-0.56)$ & $<0.001$ \\
\hline \multicolumn{3}{|l|}{ Center (reference: Center 1) } \\
\hline 2 & $1.16(0.90-1.50)$ & 0.26 \\
\hline 3 & $1.42(1.10-1.83)$ & 0.008 \\
\hline 4 & $1.78(1.33-2.39)$ & $<0.001$ \\
\hline 5 & $1.61(1.14-2.27)$ & 0.007 \\
\hline 6 & $2.24(1.53-3.28)$ & $<0.001$ \\
\hline 7 & $1.61(1.20-2.16)$ & 0.002 \\
\hline 8 & $1.81(1.34-2.43)$ & $<0.001$ \\
\hline 9 & $2.17(1.67-2.82)$ & $<0.001$ \\
\hline 10 & $2.97(2.20-4.01)$ & $<0.001$ \\
\hline 11 & $3.07(2.26-4.17)$ & $<0.001$ \\
\hline 12 & $5.46(4.03-7.39)$ & $<0.001$ \\
\hline 13 & $7.36(5.18-10.46)$ & $<0.001$ \\
\hline \multicolumn{3}{|l|}{ Infant characteristics } \\
\hline \multicolumn{3}{|l|}{$\begin{array}{l}\text { Gestational age, weeks } \\
\text { (reference: 28) }\end{array}$} \\
\hline 22 & $1.81(0.96-3.41)$ & 0.07 \\
\hline 23 & $2.54(1.86-3.47)$ & $<0.001$ \\
\hline 24 & $1.98(1.54-2.55)$ & $<0.001$ \\
\hline 25 & $1.74(1.38-2.20)$ & $<0.001$ \\
\hline 26 & $1.40(1.11-1.76)$ & 0.004 \\
\hline 27 & $1.06(0.83-1.34)$ & 0.66 \\
\hline Small for gestational age & $1.65(1.35-2.01)$ & $<0.001$ \\
\hline Male & $1.15(1.03-1.29)$ & 0.02 \\
\hline 5 -min Apgar $<5$ & $1.38(1.19-1.61)$ & $<0.001$ \\
\hline $\begin{array}{l}\text { Mechanically ventilated } \\
\text { for entire first } 7 \text { days of } \\
\text { life }\end{array}$ & $1.79(1.57-2.05)$ & $<0.001$ \\
\hline \multicolumn{3}{|l|}{ Maternal characteristics } \\
\hline Black race & $1.24(1.08-1.41)$ & 0.002 \\
\hline Multiple birth & $0.90(0.78-1.04)$ & 0.16 \\
\hline Hypertension & $0.78(0.67-0.91)$ & 0.001 \\
\hline Antenatal steroids & $0.82(0.66-1.02)$ & 0.07 \\
\hline Antenatal antibiotics & $1.18(1.01-1.37)$ & 0.03 \\
\hline $\begin{array}{l}\text { Rupture of membranes } \\
>24 \mathrm{~h}\end{array}$ & $1.84(1.59-2.14)$ & $<0.001$ \\
\hline Antepartum hemorrhage & $0.83(0.71-0.97)$ & 0.02 \\
\hline Cesarean section & $0.84(0.73-0.97)$ & 0.02 \\
\hline
\end{tabular}

without a corresponding statistically significant rise in subsequent mortality, LOS, or NEC is clinically significant.

One important issue raised by our study is whether there is a critical level, duration, or timing of antibiotic exposure at which antibiotics administered in the absence of proven infection begin to cause harm. In contrast to prior findings from the NRN and other centers, ${ }^{6,21}$ we found no significant association between prolonged early antibiotics and NEC. Multiple practice differences over the past 20 years may have contributed to this finding, including changes in the use of mothers' own milk, human donor milk, and probiotic supplements, and we did not collect detailed information on those factors. Our findings do suggest, however, that the association of empiric antibiotic use and risk of NEC has declined as overall antibiotic use has declined. The multicenter CNN study also suggests that the magnitude of antibiotic exposure may be associated with the magnitude of antibioticrelated harm. ${ }^{18}$ Increased exposure to antibiotics over an infant's entire hospital stay was associated with increased adjusted odds of a composite outcome of mortality and major morbidities, with the greatest risk associated with the highest quartile of antibiotic exposure. The prior NRN study noted increased odds of death per day of antibiotic therapy (OR $=1.16$ [95\% Cl: 1.08-1.24]), and a substantial proportion of infants received therapy for $\geq 10$ days. ${ }^{5}$ In the current study, neither duration of antibiotics nor daily antibiotic exposure data were recorded, and it is possible that our findings reflect an even larger magnitude of decreased antibiotic use than we have measured.

We continue to find substantial center variation in the use of prolonged early antibiotics (range of 30-69\% from 2008 to 2014 compared to $27-85 \%$ in 1998-2001). This variation appeared to be unrelated to the incidence of EOS at each site. It is unlikely to be due to a corresponding variation in severity of illness, as each NRN site is an academic, tertiary care center caring for relatively large numbers of extremely premature infants. Schulman and colleagues also found wide variation in California NICU antibiotic use unexplained by variation in proven infection, NEC, surgical volume, or mortality. ${ }^{22}$ We speculate that center variation in this early postnatal clinical practice represents true elective practice differences that reflect provider uncertainty regarding the risks and benefits of antibiotics administered in the absence of proven infection. In our cohort, simply being born at one academic center instead of another could increase an infant's odds of receiving prolonged early antibiotics sevenfold, even after adjustment for demographic and clinical factors. Our findings underscore an imperative for individual centers to track antibiotic utilization and to compare such data with other institutions. Robust mechanisms for sharing and receiving such data, including membership in voluntary neonatal networks, will facilitate efforts to improve local practices.

Potentially unjustified variation may matter. While not statistically significant, receipt of prolonged early antibiotics was associated with marginally increased odds of death in our cohort of infants (aOR 1.17 [95\% Cl: 0.99-1.40]). An OR $>1.0$ is consistent with the previous NRN report, which showed an aOR of 1.46 (95\% $\mathrm{Cl}$ : $1.19-1.78)$ for prolonged therapy. ${ }^{5}$ Although our analysis cannot fully account for differences in severity of illness among infants who do and do not receive prolonged early antibiotics, the potential for mediating harm remains of concern. The association between prolonged early antibiotics and fungal LOS in survivors to discharge may be an example of harm rather than confounding by indication. In this instance, relative harm was associated with prolonged early antibiotic administration to infants who were clearly less severely ill (those whose caregivers did not decide to use cMV) compared to those who were more severely ill (those who were treated with $\mathrm{cMV}$ ). Broad-spectrum antibiotic use has been previously associated with invasive candidiasis in premature infants, ${ }^{23,24}$ although why such use might differentially affect those of less illness severity, defined by available data describing degree of respiratory support, remains unclear. We speculate that less severely ill infants had fewer risk factors for fungal LOS and thus risk was increased significantly due to the use of prolonged antibiotics; more severely ill infants may have already had maximized risk of fungal LOS due to other factors. Increased perinatal and postnatal exposure to antibiotics in premature 


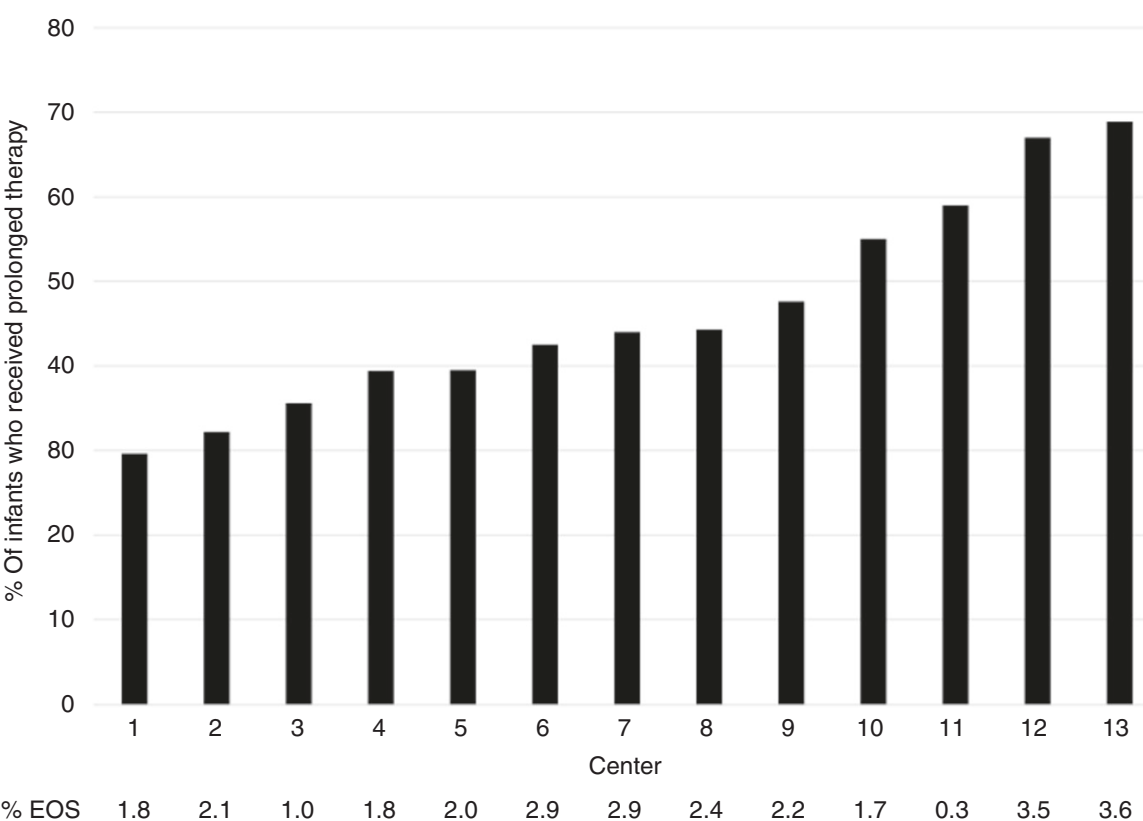

Fig. 1 Percentage of study infants receiving prolonged early antibiotic therapy and percentage of infants with early-onset sepsis (EOS) by center

\begin{tabular}{|c|c|c|c|c|}
\hline Death before discharge & 466 (19) & 409 (13) & $1.17(0.99-1.40)$ & 0.07 \\
\hline Death or NEC & $623(25)$ & $635(20)$ & $1.08(0.93-1.25)$ & 0.34 \\
\hline Death or non-CoNS LOS & $662(26)$ & $677(21)$ & $1.01(0.87-1.17)$ & 0.89 \\
\hline Death, NEC, or LOS & $1086(43)$ & $1204(38)$ & $0.97(0.86-1.11)$ & 0.66 \\
\hline $\begin{array}{l}\text { Infants who survived to } \\
\text { discharge }\end{array}$ & 2055 & 2787 & & \\
\hline NEC & $157(8)$ & $225(8)$ & $0.91(0.72-1.15)$ & 0.43 \\
\hline LOS & $540(26)$ & $670(24)$ & $0.90(0.77-1.05)$ & 0.18 \\
\hline Non-cMV group & $21(2)$ & $8(0.4)$ & $4.95(1.93-12.69)$ & $<0.001$ \\
\hline
\end{tabular}

infants has been associated with alterations in the gut microbiome, and may provide some insight into the biologic mechanisms by which prolonged antibiotic administration may be harmful in the absence of confirmed infection. ${ }^{25,26}$ It is possible that dysbiosis leads to proliferation of potential pathogens and predisposition to later infection, with resulting increases in morbidity and mortality. ${ }^{27}$ In addition, antibiotic exposure has been associated with a reduction in gastric bacterial colonization, which could increase risk for infection and increase feeding intolerance. ${ }^{28}$ Regardless of the potential mechanisms of harm or magnitude of harm, our findings support ongoing efforts to limit empiric antibiotic administration to ELBW infants at highest risk of infection and to spare those who may be at lowest risk of EOS, such as those born by cesarean section, without labor or maternal chorioamnionitis. $^{29}$

The strengths of our study include the large sample size of extremely preterm infants, uniform definitions and data collection over time, and observation of trends over time and across multiple regions in the United States. The retrospective analysis limits our ability to measure variables other than those routinely collected in the NRN database. We attempted to account for clinical severity by adjusting for mechanical ventilation, but we were unable to calculate validated severity scores such as the CRIB II or SNAPPEII. ${ }^{30,31}$ While we excluded infants with culture-confirmed sepsis in 
the first 5 postnatal days, we were unable to identify infants who may have had other true infections, such as neonatal pneumonia. We did not have data regarding the type or timing of quality improvement interventions or antibiotic stewardship programs at participating centers. Finally, while our study was similar to the previous NRN study, several differences limit comparisons between the two reports. The previous study included infants diagnosed with NEC or SIP in the first 5 days after birth, while we excluded these infants (Supplemental Digital Content S1). Our rationale was that antibiotic administration to those infants is not discretionary, and infants with NEC and SIP are likely to justifiably receive prolonged early antibiotics and have increased likelihood of mortality and morbidity. The previous NRN study included expanded data collection that detailed the exact days of exposure and the type of antibiotics prescribed on each day. This level of detail was not available to the current study. In the earlier NRN study, even 4 days of early antibiotics was associated with NEC, death, as well as the composite outcome; but in the current study, we were unable to examine the effect of antibiotics administered for $>48 \mathrm{~h}$ but $<5$ days, and the risk of this practice remains unclear. We also do not know what proportion of infants had no antibiotics given in the first $72 \mathrm{~h}$ after birth $(\sim 2-3 \%$ had none given in the prior study), ${ }^{5}$ and we do not know what proportion of infants were administered cephalosporins, antibiotics that are associated with higher mortality and Candida infections. ${ }^{24,32}$

\section{CONCLUSION}

In this study of 5730 infants across 13 NRN centers, the proportion of infants who received prolonged early antibiotics in the absence of EOS, NEC, or SIP decreased significantly between 2008 and 2014. Overall prolonged antibiotic use also declined in comparison to a prior NRN study that included infants born between 1998 and 2001. Prolonged early antibiotic exposure continues to vary widely across NRN centers. Our findings support ongoing efforts to promote neonatal antibiotic stewardship, as supported by the Centers for Disease Control and Prevention. ${ }^{33}$ Such efforts must include robust means of identifying those infants who will derive maximum benefit and minimum harm from antibiotic treatments that will be acceptable across different sites of neonatal care.

\section{ACKNOWLEDGEMENTS}

The National Institutes of Health and the Eunice Kennedy Shriver National Institute of Child Health and Human Development (NICHD) provided grant support for the Neonatal Research Network's Generic Database Study (high-risk registry) through cooperative agreements. While NICHD staff did have input into the study design, conduct, analysis, and manuscript drafting, the comments and views of the authors do not necessarily represent the views of the NICHD.

Data collected at participating sites of the NICHD Neonatal Research Network (NRN) were transmitted to RTI International, the data coordinating center (DCC) for the network, which stored, managed, and analyzed the data for this study. On behalf of the NRN, Dr. Abhik Das (DCC Principal Investigator) and Ms. Dhuly Chowdhury and Ms. Nellie Hansen (DCC Statisticians) had full access to all the data in the study and take responsibility for the integrity of the data and accuracy of the data analysis. The sites with data included in this study are denoted with an asterisk.

We are indebted to our medical and nursing colleagues and the infants and their parents who agreed to take part in this study. The following investigators, in addition to those listed as authors, participated in this study:

NRN Steering Committee Chair: Michael S. Caplan, MD, University of Chicago, Pritzker School of Medicine; Richard A. Polin, MD, Division of Neonatology, College of Physicians and Surgeons, Columbia University (2011-present).

*Alpert Medical School of Brown University and Women \& Infants Hospital of Rhode Island (U10 HD27904) - Abbot R. Laptook, MD; Martin Keszler, MD; Angelita M. Hensman, MS RNC-NIC; Kristin M. Basso, MaT RN; Elisa Vieira, RN BSN; Emily Little, BSN RN.

*Case Western Reserve University, Rainbow Babies \& Children's Hospital (U10 HD21364, M01 RR80)-Michele C. Walsh, MD MS; Avroy A. Fanaroff, MD; Anna Marie Hibbs, MD; Nancy S. Newman, BA RN; Bonnie S. Siner, RN.
Children's Mercy Hospital, University of Missouri Kansas City School of Medicine (U10 HD68284)—William E. Truog, MD; Howard W. Kilbride, MD; Eugenia K. Pallotto, MD MSCE; Cheri Gauldin, RN BSN CCRC; Anne Holmes RN MSN MBA-HCM CCRC; Kathy Johnson RN, CCRC; Allison Knutson, BSN RNC-NIC.

*Cincinnati Children's Hospital Medical Center, University Hospital, and Good Samaritan Hospital (U10 HD27853, M01 RR8084)—Brenda B. Poindexter, MD MS; Kurt Schibler, MD; Barbara Alexander, RN; Cathy Grisby, BSN CCRC; Lenora D. Jackson, CRC; Kristin Kirker, CRC; Greg Muthig, BS.

*Duke University School of Medicine, University Hospital, University of North Carolina, and Duke Regional Hospital (U10 HD40492, M01 RR30, UL1 TR83)-Ronald N. Goldberg, MD; Kimberley A. Fisher, PhD FNP-BC IBCLC; Sandra Grimes, RN BSN; Joanne Finkle, RN JD; Matthew M. Laughon, MD MPH; Carl L. Bose, MD; Janice Bernhardt, MS RN; Gennie Bose, RN.

*Emory University, Children's Healthcare of Atlanta, Grady Memorial Hospital, and Emory University Hospital Midtown (U10 HD27851, UL1 TR454)—David P. Carlton, MD; Ellen C. Hale, RN BS CCRC; Ann Blackwelder, RN MSN; Yvonne C. Loggins, RN BSN; Diane I. Bottcher, RN MSN.

Eunice Kennedy Shriver National Institute of Child Health and Human DevelopmentStephanie Wilson Archer, MA.

*Indiana University, University Hospital, Methodist Hospital, Riley Hospital for Children, and Wishard Health Services (U10 HD27856, M01 RR750, UL1 TR6)—Brenda B. Poindexter, MD MS; Gregory M. Sokol, MD; Leslie Dawn Wilson, BSN CCRC; Dianne E. Herron, RN CCRC.

*McGovern Medical School at The University of Texas Health Science Center at Houston, Children's Memorial Hermann Hospital (U10 HD21373)-Kathleen A. Kennedy, MD MPH; Jon E. Tyson, MD MPH; Julie Arldt-McAlister, MSN APRN; Katrina Burson, RN BSN; Carmen Garcia, RN BSN CCRP; Beverly Foley Harris, RN BSN; Karen Martin, RN; Sara C. Martin, RN BSN; Georgia E. McDavid, RN; Shawna Rodgers, RN BSN; Patti L. Pierce Tate, RCP; Sharon L. Wright, MT (ASCP).

Nationwide Children's Hospital and the Ohio State University Medical Center (U10 HD68278)—Leif D. Nelin, MD; Sudarshan R. Jadcherla, MD; Patricia Luzader, RN; Christine A. Fortney, PhD RN; Gail E. Besner; Nehal A. Parikh, MD.

RTI International (U10 HD36790)—Dennis Wallace, PhD; Marie G. Gantz, PhD; Jeanette O'Donnell Auman, BS; Margaret M. Crawford, BS CCRP; Jenna Gabrio, MPH CCRP; Carolyn M. Petrie Huitema, MS CCRP; Kristin M. Zaterka-Baxter, RN BSN CCRP.

*Stanford University, Dominican Hospital, El Camino Hospital, and Lucile Packard Children's Hospital (U10 HD27880, M01 RR70, UL1 TR93)—Krisa P. Van Meurs, MD; Marian M. Adams, MD; David K. Stevenson, MD; M. Bethany Ball, BS CCRC; Andrew W. Palmquist, RN BSN; Melinda S. Proud, RCP.

Tufts Medical Center, Floating Hospital for Children (U10 HD53119, M01 RR54)_Ivan D. Frantz III, MD; John M. Fiascone, MD; Brenda L. MacKinnon, RNC; Ellen Nylen, RN BSN.

*University of Alabama at Birmingham Health System and Children's Hospital of Alabama (U10 HD34216, M01 RR32)-Waldemar A. Carlo, MD; Namasivayam Ambalavanan, MD; Monica V. Collins, RN BSN MaEd; Shirley S. Cosby, RN BSN. University of California-Los Angeles, Mattel Children's Hospital, Santa Monica Hospital, Los Robles Hospital and Medical Center, and Olive View Medical Center (U10 HD68270) - Uday Devaskar, MD; Meena Garg, MD; Teresa Chanlaw, MPH; Rachel Geller, RN BSN.

*University of lowa and Mercy Medical Center (U10 HD53109, M01 RR59)—Edward F. Bell, MD; Dan L. Ellsbury, MD; John A. Widness, MD; Tarah T. Colaizy, MD MPH; Karen J. Johnson, RN BSN; Donia B. Campbell, RNC-NIC; Jacky R. Walker, RN.

*University of New Mexico Health Sciences Center (U10 HD53089, UL1 TR41)—Kristi L. Watterberg, MD; Robin K. Ohls, MD; Conra Backstrom Lacy, RN; Rebecca A. Tomson, BSN RNC; Carol Hartenberger, BSN MPH; Sandra Sundquist Beauman, MSN RNC-NIC. University of Pennsylvania, Hospital of the University of Pennsylvania, Pennsylvania Hospital, and Children's Hospital of Philadelphia (U10 HD68244)—Barbara Schmidt, MD MSc; Haresh Kirpalani, MB MSc; Sara B. DeMauro, MD MSCE; Kevin C. Dysart, MD; Aasma S. Chaudhary, BS RRT; Soraya Abbasi, MD; Toni Mancini, RN BSN CCRC; Dara M. Cucinotta, RN; Erik A. Jensen, MD MSCE.

University of Rochester Medical Center, Golisano Children's Hospital, and the University of Buffalo Women's and Children's Hospital of Buffalo (U10 HD68263, M01 RR44, UL1 TR42)—Carl T. D'Angio, MD; Ronnie Guillet, MD PhD; Satyan Lakshminrusimha, MD; Anne Marie Reynolds, MD, MPH; Linda J. Reubens, RN CCRC; Rosemary Jensen; Deana Maffett, RN; Holly I.M. Wadkins, MA; Michael G. Sacilowski, MAT; Ashley Williams, MS Ed; Stephanie Guilford, BS; Mary Rowan, RN; Diane M. Prinzing, AAS; Julianne Hunn, BS; Ann Marie Scorsone, MS CCRC; Karen Wynn, RN; Melissa Bowman, RN; Dale L. Phelps, MD; Aimee Horan, LPN.

*University of Texas Southwestern Medical Center at Dallas, Parkland Health \& Hospital System, and Children's Medical Center Dallas (U10 HD40689, M01 RR633) Myra H. Wyckoff, MD; Pablo J. Sánchez, MD; Luc P. Brion, MD; Lijun Chen, PhD RN; Alicia Guzman; Janet S. Morgan, RN; Lara Pavageau, MD; Diana M. Vasil, MSN BSN RNC-NIC; Lizette E. Torres, RN.

University of Utah University Hospital, Intermountain Medical Center, LDS Hospital, and Primary Children's Medical Center (U10 HD53124, M01 RR64, UL1 TR105)—Roger 
G. Faix, MD; Bradley A. Yoder, MD; Karen A. Osborne, RN BSN CCRC; Karie Bird, RN BSN; Jill Burnett, RNC BSN; Jennifer J. Jensen, RN BSN; Cynthia Spencer, RNC BSN; Kimberlee Weaver-Lewis, RN MS; Karen Zanetti, RN.

*Wayne State University, University of Michigan, Hutzel Women's Hospital, and Children's Hospital of Michigan (U10 HD21385)—Seetha Shankaran, MD; John Barks, MD; Rebecca Bara, RN BSN; Mary Johnson, RN BSN; Mary Christensen, RT; Stephanie Wiggins, MS

Yale University, Yale-New Haven Children's Hospital, and Bridgeport Hospital (U10 HD27871, UL1 TR142)-Richard A. Ehrenkranz, MD; Harris Jacobs, MD; Patricia Cervone, RN; Monica Konstantino, RN BSN; JoAnn Poulsen, RN; Janet Taft, RN BSN.

\section{AUTHOR CONTRIBUTIONS}

R.G.G. conceptualized and designed the study, drafted the initial manuscript, interpreted the data analyses, and reviewed and revised the manuscript. D.C., N.I.H., and A.D. carried out the data analysis, assisted with interpretation of the data analyses, and reviewed and revised the manuscript for important intellectual content. C.M.C. assisted with acquisition of the data, interpreted the data analyses, reviewed and revised the manuscript for important intellectual content, and obtained funding to support the study. B.J.S. assisted with acquisition of data and critical revision of the manuscript for important intellectual content. R.D.H. conceptualized and designed the study, assisted with data analysis and interpretation, and provided critical revision of the manuscript for intellectual content. P.B.S., K.M.P., and S.M. provided analysis and interpretation of the data and critical revision of the manuscript for important intellectual content. P.J.S. conceptualized and designed the study, assisted with acquisition of data, provided analysis and interpretation of the data, and obtained funding for support of the study. All authors approved the final manuscript as submitted and agree to be accountable for all aspects of the work.

\section{ADDITIONAL INFORMATION}

The online version of this article (https://doi.org/10.1038/s41390-019-0300-4) contains supplementary material, which is available to authorized users.

Competing interests: The authors declare no competing interests.

Publisher's note: Springer Nature remains neutral with regard to jurisdictional claims in published maps and institutional affiliations.

\section{REFERENCES}

1. Hsieh, E. M. et al. Medication use in the neonatal intensive care unit. Am. J. Perinatol. 31, 811-821 (2014).

2. Schrag, S. J. et al. Epidemiology of invasive early-onset neonatal sepsis, 2005 to 2014. Pediatrics 138, e20162013 (2016). pii.

3. Kumar, Y. et al. Time to positivity of neonatal blood cultures. Arch. Dis. Child. Fetal Neonatal Ed. 85, F182-F186 (2001).

4. Jardine, L., Davies, M. W. \& Faoagali, J. Incubation time required for neonatal blood cultures to become positive. J. Paediatr. Child Health 42, 797-802 (2006).

5. Cotten, C. M. et al. Prolonged duration of initial empirical antibiotic treatment is associated with increased rates of necrotizing enterocolitis and death for extremely low birth weight infants. Pediatrics 123, 58-66 (2009).

6. Kuppala, V. S. et al. Prolonged initial empirical antibiotic treatment is associated with adverse outcomes in premature infants. J. Pediatr. 159, 720-725 (2011).

7. Cordero, L. \& Ayers, L. W. Duration of empiric antibiotics for suspected early-onset sepsis in extremely low birth weight infants. Infect. Control. Hosp. Epidemiol. 24, 662-666 (2003).

8. Flannery, D. D. et al. Temporal trends and center variation in early antibiotic use among premature infants. JAMA Netw. Open 1, e180164 (2018).

9. Alexander, G. R. et al. A United States national reference for fetal growth. Obstet. Gynecol. 87, 163-168 (1996).

10. Bell, M. J. Neonatal necrotizing enterocolitis. N. Engl. J. Med. 298, 281-282 (1978).
11. Walsh, M. C. \& Kliegman, R. M. Necrotizing enterocolitis: treatment based on staging criteria. Pediatr. Clin. North Am. 33, 179-201 (1986).

12. Society for Healthcare Epidemiology of America, Infectious Diseases Society of America, Pediatric Infectious Diseases Society. Policy statement on antimicrobial stewardship by the Society for Healthcare Epidemiology of America (SHEA), the Infectious Diseases Society of America (IDSA), and the Pediatric Infectious Diseases Society (PIDS). Infect. Control. Hosp. Epidemiol. 33, 322-327 (2012).

13. Vermont Oxford Network. https://public.vtoxford.org/quality-education/inicq2018. Accessed 2018.

14. Flett, K. B. et al. Impact of mandatory public reporting of central line-associated bloodstream infections on blood culture and antibiotic utilization in pediatric and neonatal intensive care units. Infect. Control. Hosp. Epidemiol. 36, 878-885 (2015).

15. Zingg, W. et al. Secular trends in antibiotic use among neonates: 2001-2008. Pediatr. Infect. Dis. J. 30, 365-370 (2011).

16. Makri, V. et al. Managing antibiotics wisely: a quality improvement programme in a tertiary neonatal unit in the UK. BMJ Open Qual. 7, e000285 (2018).

17. Dukhovny, D. Antimicrobial stewardship in neonatal intensive care: The Oregon and Southwest Washington Collaboration. https://blogs.cdc.gov/safehealthcare/ antimicrobial-stewardship-in-neonatal-intensive-care-the-oregon-and-southwestwashington-collaboration/. Accessed 2018.

18. Ting, J. Y. et al. Association between antibiotic use and neonatal mortality and morbidities in very low-birth-weight infants without culture-proven sepsis or necrotizing enterocolitis. JAMA Pediatr. 170, 1181-1187 (2016).

19. Ting, J. Y. et al. Reduction of inappropriate antimicrobial prescriptions in a tertiary neonatal intensive care unit following antimicrobial stewardship care bundle implementation. Pediatr. Infect. Dis. J. https://doi.org/10.1097/INF.0000000000002039 (2018).

20. Nzegwu, N. I. et al. Implementation of an antimicrobial stewardship program in a neonatal intensive care unit. Infect. Control. Hosp. Epidemiol. 38, 1137-1143 (2017).

21. Abdel Ghany, E. A. \& Ali, A. A. Empirical antibiotic treatment and the risk of necrotizing enterocolitis and death in very low birth weight neonates. Ann. Saudi. Med. 32, 521-526 (2012).

22. Schulman, J. et al. Neonatal intensive care unit antibiotic use. Pediatrics 135, 826-833 (2015).

23. Benjamin, D. K. et al. Neonatal candidiasis: epidemiology, risk factors, and clinical judgment. Pediatrics 126, e865-e873 (2010).

24. Cotten, C. M. et al. The association of third-generation cephalosporin use and invasive candidiasis in extremely low birth-weight infants. Pediatrics 118, 717-722 (2006).

25. Arboleya, S. et al. Intestinal microbiota development in preterm neonates and effect of perinatal antibiotics. J. Pediatr. 166, 538-544 (2015).

26. Dardas, M. et al. The impact of postnatal antibiotics on the preterm intestinal microbiome. Pediatr. Res. 76, 150-158 (2014).

27. Greenwood, C. et al. Early empiric antibiotic use in preterm infants is associated with lower bacterial diversity and higher relative abundance of Enterobacter. $J$. Pediatr. 165, 23-29 (2014).

28. Patel, K. et al. Trends and determinants of gastric bacterial colonization of preterm neonates in a NICU setting. PLoS ONE 10, e0114664 (2015).

29. Puopolo, K. M. et al. Identification of extremely premature infants at low risk for early-onset sepsis. Pediatrics 140, e20170925 (2017).

30. Parry, G., Tucker, J. \& Tarnow-Mordi, W. UK Neonatal Staffing Study Collaborative Group. CRIB II: an update of the clinical risk index for babies score. Lancet 361, 1789-1791 (2003).

31. Richardson, D. K. et al. SNAP-II and SNAPPE-II: simplified newborn illness severity and mortality risk scores. J. Pediatr. 138, 92-100 (2001).

32. Clark, R. H. et al. Empiric use of ampicillin and cefotaxime, compared with ampicillin and gentamicin, for neonates at risk for sepsis is associated with an increased risk of neonatal death. Pediatrics 117, 67-74 (2006).

33. Centers for Disease Control and Prevention. Core elements of hospital antibiotic stewardship programs. https://www.cdc.gov/antibiotic-use/healthcare/ implementation/core-elements.html. 